\title{
CONTROL IMPROVEMENT FOR JUMP-DIFFUSION PROCESSES WITH APPLICATIONS TO FINANCE
}

\author{
NICOLE BÄUERLE* AND ULRICH RIEDER ${ }^{\ddagger}$
}

\begin{abstract}
We consider stochastic control problems with jump-diffusion processes and formulate an algorithm which produces, starting from a given admissible control $\pi$, a new control with a better value. If no improvement is possible, then $\pi$ is optimal. Such an algorithm is well-known for discrete-time Markov Decision Problems under the name Howard's policy improvement algorithm. The idea can be traced back to Bellman. Here we show with the help of martingale techniques that such an algorithm can also be formulated for stochastic control problems with jump-diffusion processes. As an application we derive some interesting results in portfolio optimization.
\end{abstract}

\section{AMS 2010 Classification: 93E20, 60J75, 91G10}

\section{INTRODUCTION}

For discrete-time Markov control problems (also known under the name Markov decision problems) there exists a well-known algorithm which produces, starting from a given admissible control $\pi$, a new control with a better value. If no improvement is possible, then $\pi$ is optimal. This algorithm is known under the name Howard's policy improvement algorithm and has been proposed by Howard (see Howard (1960)). In this paper now we consider continuous-time stochastic control problems for jump-diffusion processes and formulate a corresponding improvement algorithm. The idea is rather general and holds in principle for a large class of control problems including controlled Lévy processes, given some technical conditions are satisfied. Indeed related ideas can be found in Fleming and Rishel (1975) p. 168 (in the case of diffusion processes) and Schmidli (2008) p.48 for showing the existence of solutions of HJB equations. But both do not use the concept to improve controls or characterize optimal policies. In order to keep the exposition simple we restrict here to controlled jump-diffusions. Still in this case for the general problem the usual continuity and growth conditions have to be satisfied, so it is often easier to apply the algorithm to specific problems. For the theory of general stochastic control problems see e.g. Fleming and Soner (1993), Yong and Zhou (1999), Kushner and Dupuis (2001) or Øksendal and Sulem (2005).

The algorithm can be used in different ways: First it can be used to improve a naive control which arises from ad hoc considerations. Second it can be used to identify conditions under which a certain control is optimal.

The paper is organized as follows: In the next section we introduce the finite horizon stochastic control problem and a number of technical assumptions. In 
Section 3 we present the improvement algorithm and show its properties. We also shortly investigate the infinite horizon problem which is not much different. In the last section we give some applications from portfolio optimization. We consider a jump-diffusion financial market and show that the "invest all the money in the bond" strategy is optimal if and only if the drift of the stock is the same as the drift of the bond. Moreover, we prove that the CRRA-utility functions are the only utility functions $U:(0, \infty) \rightarrow \mathbb{R}$ with $U \in C^{2}$ where the optimal portfolio invests a constant fraction of the wealth in the stock.

\section{The ModeL}

The method we present here is quite general and we have decided to explain it in the framework of controlled jump-diffusion problems for the sake of readability. All appearing processes are defined on the filtered probability space $\left(\Omega, \mathcal{F},\left(\mathcal{F}_{t}\right), P\right)$. In what follows $W=\left(W_{1}, \ldots, W_{m}\right)$ is an $m$-dimensional Brownian motion and $N=\left(N_{1}, \ldots, N_{l}\right)$ are independent homogeneous Poisson random measures w.r.t. $\left(\mathcal{F}_{t}\right)$. More precisely $N_{j}(t, B)$ is the number of jumps of process $j$ with size in $B \in$ $\mathcal{B}, 0 \notin B$ which occur up to time $t$. Let $\nu_{j}(B)=\mathbb{E} N_{j}(1, B)$ be the corresponding Lévy measure and

$$
\tilde{N}_{j}\left(d t, d z_{j}\right)=N_{j}\left(d t, d z_{j}\right)-\nu_{j}\left(d z_{j}\right) d t
$$

the compensated Poisson random measure. The $n$-dimensional controlled state process $X=\left(X_{1}, \ldots, X_{n}\right)$ is given by

$$
\begin{aligned}
d X_{i}(t)= & \mu_{i}\left(t, X_{t}, \pi_{t}\right) d t+\sum_{j=1}^{m} \sigma_{i j}\left(t, X_{t}, \pi_{t}\right) d W_{j}(t)+ \\
& +\sum_{j=1}^{l} \int \gamma_{i j}\left(t, X_{t-}, \pi_{t-}, z_{j}\right) \tilde{N}_{j}\left(d t, d z_{j}\right)
\end{aligned}
$$

for $i=1, \ldots, n$ where $\pi=\left(\pi_{t}\right)$ is a càdlàg $\left(\mathcal{F}_{t}\right)$-adapted control process with values in $D \subset \mathbb{R}^{d}$. We say that $\pi$ is admissible and write $\pi \in A(t, x)$ if $X_{t}=x$ and (2.1) has a unique strong solution. In this section we will investigate a control problem with finite time horizon $T>0$. Let us denote $E:=[0, T] \times \mathbb{R}^{n}$. The coefficient functions are given by $\mu: E \times D \rightarrow \mathbb{R}^{n}, \sigma: E \times D \rightarrow \mathbb{R}^{n \times m}, \gamma_{i j}: E \times D \times \mathbb{R} \rightarrow \mathbb{R}$ and are supposed to be continuous in $t$ and $x$. We will also use the notation $\gamma^{(j)}:=$ $\left(\gamma_{1 j}, \ldots, \gamma_{n j}\right), j=1, \ldots, l$. The function we want to maximize is for $\pi \in A(t, x)$ given by

$$
J^{\pi}(t, x):=\mathbb{E}_{t, x}\left[\int_{t}^{T} g\left(s, X_{s}, \pi_{s}\right) d s+h\left(X_{T}\right)\right], \quad(t, x) \in E
$$

where $\mathbb{E}_{t, x}[\cdot]=\mathbb{E}\left[\cdot \mid X_{t}=x\right]$ and $g: E \times D \rightarrow \mathbb{R}$ is the running reward and $h: \mathbb{R}^{n} \rightarrow \mathbb{R}$ the terminal reward. Both functions are assumed to be continuous. Note that $J^{\pi}(T, x)=h(x)$ for all $\pi$. The value function is denoted by

$$
J(t, x)=\sup _{\pi \in A(t, x)} J^{\pi}(t, x), \quad(t, x) \in E .
$$

It is well-known (see Øksendal and Sulem (2005)) that under some mild conditions the optimal control $\pi^{*}=\left(\pi_{t}^{*}\right)$ can be found among the Markov controls, i.e. there exists a feedback function $\pi^{*}: E \rightarrow D$ such that $\pi_{t}^{*}=\pi^{*}\left(t, X_{t}^{*}\right)$ where $X^{*}$ is the corresponding state process. With an abuse of notation we will write $\pi$ for the 
control process as well as for the feedback function. The correct notion should always be clear from the context. We assume that

$$
\sup _{\pi \in A(t, x)} \mathbb{E}_{t, x}\left[\int_{t}^{T}\left|g\left(s, X_{s}, \pi_{s}\right)\right| d s+\left|h\left(X_{T}\right)\right|\right]<\infty .
$$

The generator of the state process is for $v \in C^{1,2}$ and $(t, x) \in E, u \in D$ given by

$$
\begin{aligned}
& \mathcal{A} v(t, x, u):=v_{t}(t, x)+\sum_{i=1}^{n} v_{x_{i}}(t, x) \mu_{i}(t, x, u)+\frac{1}{2} \sum_{i, j=1}^{n}\left(\sigma \sigma^{T}\right)_{i j}(t, x, u) v_{x_{i} x_{j}}(t, x)+ \\
& +\sum_{j=1}^{l} \int\left(v\left(t, x+\gamma^{(j)}\left(t, x, u, z_{j}\right)\right)-v(t, x)-\nabla_{x} v(t, x) \gamma^{(j)}\left(t, x, u, z_{j}\right)\right) \nu_{j}\left(d z_{j}\right) .
\end{aligned}
$$

Let $\pi, \hat{\pi} \in A(t, x)$ and suppose $\hat{X}$ is the state process which belongs to $\hat{\pi}$. The following assumption is important in applying the Dynkin formula later:

$$
\begin{aligned}
& \mathbb{E}_{t, x}\left[h\left(\hat{X}_{T}\right)+\int_{0}^{T}\left\|\mathcal{A} J^{\pi}\left(s, \hat{X}_{s}, \hat{\pi}_{s}\right)\right\|+\left\|\sigma^{T}\left(s, \hat{X}_{s}, \hat{\pi}_{s}\right) \nabla_{x} J^{\pi}\left(s, \hat{X}_{s}\right)\right\|^{2}+\right. \\
& \left.+\int \sum_{j=1}^{k}\left|J^{\pi}\left(s, \hat{X}_{s}+\gamma^{(j)}\left(s, \hat{X}_{s}, \hat{\pi}_{s}, z_{j}\right)\right)-J^{\pi}\left(s, \hat{X}_{s}\right)\right|^{2} \nu_{j}\left(d z_{j}\right) d s\right]<\infty .
\end{aligned}
$$

We will refer to it when necessary.

\section{Improvement of Controls}

Given an admissible control $\pi$, the following algorithm constructs a new control $\hat{\pi}$ with a higher value and if an improvement is not possible, then we can conclude that $\pi$ is optimal. The algorithm is as follows:

\section{Control Improvement Algorithm:}

(a) Suppose $\pi$ is an admissible control and the corresponding value function satisfies $J^{\pi} \in C^{1,2}(E)$.

(b) Define a new feedback function $\hat{\pi}(t, x)$ such that it maximizes

$$
u \mapsto g(t, x, u)+\mathcal{A} J^{\pi}(t, x, u), \quad u \in D
$$

for all $(t, x) \in E$ and suppose that $\hat{\pi}(t, x)$ defines an admissible control $\hat{\pi}_{t}:=$ $\hat{\pi}\left(t, \hat{X}_{t}\right)$. If $\pi(t, x)$ is among the maximum points, then set $\hat{\pi}(t, x):=\pi(t, x)$.

The new control $\hat{\pi}$ has the following properties:

Theorem 3.1. Let $I:=\left\{(t, x) \in E: g(t, x, \hat{\pi}(t, x))+\mathcal{A} J^{\pi}(t, x, \hat{\pi}(t, x))>0\right\}$ and suppose $\hat{\pi}$ satisfies $(2.3)$.

a) If $I \neq \emptyset$, then $J^{\hat{\pi}}(t, x) \geq J^{\pi}(t, x)$ for all $(t, x) \in E$ and $J^{\hat{\pi}}(t, x)>J^{\pi}(t, x)$ for $(t, x) \in I$.

b) If $I=\emptyset$ then $\hat{\pi}$ is an optimal control.

Proof. Suppose $\pi$ is an admissible control with state process $X$ and the corresponding value function satisfies $J^{\pi} \in C^{1,2}(E)$. The process $M^{t, \pi}$ defined for $s \in[t, T]$ by

$$
M_{s}^{t, \pi}:=\int_{t}^{s} g\left(r, X_{r}, \pi_{r}\right) d r+J^{\pi}\left(s, X_{s}\right)
$$


is a martingale for all $t$ and $\pi$. This is easily seen since (2.2) holds and for $t \leq s<$ $\tau \leq T$ we obtain:

$$
J^{\pi}\left(s, X_{s}\right)=\mathbb{E}\left[\int_{s}^{\tau} g\left(r, X_{r}, \pi_{r}\right) d r+J^{\pi}\left(\tau, X_{\tau}\right) \mid \mathcal{F}_{s}\right] .
$$

An application of Itô's Lemma to the process $X$ and the function $J^{\pi}$ yields:

$$
\begin{aligned}
d M_{t}^{\tau, \pi} & =g\left(t, X_{t}, \pi_{t}\right) d t+d J^{\pi}\left(t, X_{t}\right) \\
& =g\left(t, X_{t}, \pi_{t}\right) d t+\mathcal{A} J^{\pi}\left(t, X_{t}, \pi_{t}\right) d t+J_{x}^{\pi}\left(t, X_{t}\right) \sigma\left(t, X_{t}, \pi_{t}\right) d W_{t} \\
& +\int_{\mathbb{R}} J^{\pi}\left(t, X_{t-}+\gamma\left(t, X_{t}, \pi_{t}, z\right)\right)-J^{\pi}\left(t, X_{t-}\right) \tilde{N}(d t, d z) .
\end{aligned}
$$

Since $M^{\tau, \pi}$ is a martingale we must have

$$
g(t, x, \pi(t, x))+\mathcal{A} J^{\pi}(t, x, \pi(t, x))=0
$$

for all $(t, x)$ where $\pi(t, x)$ is the feedback control of $\pi$. Next suppose $\hat{\pi}$ is obtained by the algorithm and satisfies the stated properties. In particular $\hat{X}$ is the state process derived by $\hat{\pi}$. An application of the Dynkin formula (see e.g. Øksendal and Sulem (2005) Theorem 1.24 and note that $(2.3)$ holds) to the process $\hat{X}$ and the function $J^{\pi}$ yields:

$$
\begin{aligned}
\mathbb{E}_{t, x}\left[J^{\pi}\left(T, \hat{X}_{T}\right)\right]-J^{\pi}(t, x) & =\mathbb{E}_{t, x}\left[\int_{t}^{T} \mathcal{A} J^{\pi}\left(s, \hat{X}_{s}, \hat{\pi}_{s}\right) d s\right] \\
& \geq-\mathbb{E}_{t, x}\left[\int_{t}^{T} g\left(s, \hat{X}_{s}, \hat{\pi}_{s}\right) d s\right]
\end{aligned}
$$

where the inequality follows from (3.1) and the fact that $\hat{\pi}$ maximizes this expression. Arranging terms and noting that $J^{\pi}\left(T, \hat{X}_{T}\right)=h\left(\hat{X}_{T}\right)$ we obtain:

$$
J^{\hat{\pi}}(t, x) \geq J^{\pi}(t, x), \quad(t, x) \in E .
$$

The inequality is strict if $(t, x) \in I$, since then $g(t, x, \hat{\pi}(t, x))+\mathcal{A} J^{\pi}(t, x, \hat{\pi}(t, x))>0$ and due to the continuity assumptions on $g$ and $J^{\pi}$ and its derivatives and the continuity of $t \mapsto \hat{X}_{t}$ in a small time intervall with positive probability, this inequality is also satisfied in an environment of $(t, x)$ with positive probability which yields part a).

If $I=\emptyset$, i.e. no improvement is possible, then $\hat{\pi}=\pi$. Let $\tilde{\pi} \in A(t, x)$ be an arbitrary admissible control with state process $\tilde{X}$. Since

$$
g(t, x, \tilde{\pi}(t, x))+\mathcal{A} J^{\pi}(t, x, \tilde{\pi}(t, x)) \leq 0,
$$

the process $Y^{t, \tilde{\pi}}$ defined for $s \in[t, T]$ by

$$
Y_{s}^{t, \tilde{\pi}}:=\int_{t}^{s} g\left(r, \tilde{X}_{r}, \tilde{\pi}_{r}\right) d r+J^{\pi}\left(s, \tilde{X}_{s}\right)
$$

is a supermartingale for all $t$ and $\tilde{\pi}$. Hence

$$
\begin{aligned}
J^{\pi}(t, x) & =\mathbb{E}_{t, x}\left[Y_{t}^{t, \tilde{\pi}}\right] \geq \mathbb{E}_{t, x}\left[Y_{T}^{t, \tilde{\pi}}\right] \\
& =\mathbb{E}_{t, x}\left[\int_{t}^{T} g\left(r, \tilde{X}_{r}, \tilde{\pi}_{r}\right) d r+J^{\pi}\left(T, \tilde{X}_{T}\right)\right]=J^{\tilde{\pi}}(t, x)
\end{aligned}
$$

and $\hat{\pi}=\pi$ is optimal. 
Remark 3.2. The statement in Theorem 3.1 indeed implies that $I=\emptyset$ if and only if $\hat{\pi}$ is an optimal control. This makes it possible to characterize within a parametric model the optimality of certain controls. Moreover, it is easily shown that $I=\emptyset$ if and only if $\hat{\pi}(t, x)$ maximizes

$$
u \mapsto g(t, x, u)+\mathcal{A} J(t, x, u), \quad u \in D
$$

for all $(t, x) \in E$.

Now suppose $\left(\pi^{k}\right)_{k \in \mathbb{N}}$ is a sequence of admissible controls which are generated by the algorithm satisfying (2.3) together with the corresponding value functions $\left(J^{k}\right)$ where $J^{k}:=J^{\pi^{k}}$. Since $J^{k}(t, x)$ is increasing in $k$ the limit $J^{\infty}:=\lim _{k \rightarrow \infty} J^{k}$ obviously exists. We obtain the following result:

Theorem 3.3. Suppose that the following assumptions are satisfied:

(i) $\lim _{k \rightarrow \infty} J^{k}=: J^{\infty} \in C^{1,2}$ and $J_{t}^{k} \rightarrow J_{t}^{\infty}, J_{x}^{k} \rightarrow J_{x}^{\infty}, J_{x x}^{k} \rightarrow J_{x x}^{\infty}$ uniformly on $E$ and $J^{\infty}$ satisfies (2.3) for all $\pi \in A(t, x)$.

(ii) $\mu, \sigma$ and $\gamma$ are bounded, i.e. there exists a $K>0$ such that $|\mu|,|\sigma|,|\gamma| \leq K$. Let $\pi$ be a control defined by the maximizer of $J^{\infty}$ as in step (b) of the algorithm. Then $J=J_{\infty}$ and $\pi$ is optimal.

Proof. In what follows we write $X^{k}$ instead of $X^{\pi^{k}}$. From the previous proof we know that for $(t, x) \in E$ :

$$
\begin{aligned}
J^{k}(t, x) & =J^{k-1}(t, x)+\mathbb{E}_{t, x}\left[\int_{t}^{T} g\left(s, X_{s}^{k}, \pi_{s}^{k}\right)+\mathcal{A} J^{k-1}\left(s, X_{s}^{k}, \pi_{s}^{k}\right) d s\right] \\
& =J^{k-1}(t, x)+\mathbb{E}_{t, x}\left[\int_{t}^{T} \sup _{u \in D}\left\{g\left(s, X_{s}^{k}, u\right)+\mathcal{A} J^{k-1}\left(s, X_{s}^{k}, u\right)\right\} d s\right] .
\end{aligned}
$$

Letting $k \rightarrow \infty$ we obtain

$$
\lim _{k \rightarrow \infty} \mathbb{E}_{t, x}\left[\int_{t}^{T} \sup _{u \in D}\left\{g\left(s, X_{s}^{k}, u\right)+\mathcal{A} J^{k-1}\left(s, X_{s}^{k}, u\right)\right\} d s\right]=0 .
$$

Now we claim that $J^{\infty}$ satisfies the HJB equation, i.e.

$$
\sup _{u \in D}\left\{g(t, x, u)+\mathcal{A} J^{\infty}(t, x, u)\right\}=0, \quad(t, x, u) \in E \times D
$$

which then implies the statement. This statement is shown by contradiction: Suppose there exists a $(t, x) \in E$ such that

$$
g\left(t, x, u^{*}\right)+\mathcal{A} J^{\infty}\left(t, x, u^{*}\right)=\varepsilon_{1}>0
$$

for an $u^{*} \in D$. By assumption (i) we can choose $k_{0}$ and $\varepsilon_{1} \gg \varepsilon_{2}>0$ such that for all $k \geq k_{0}$ :

$$
\max \left\{\left|J^{\infty}-J^{k}\right|,\left|J_{t}^{\infty}-J_{t}^{k}\right|,\left|J_{x}^{\infty}-J_{x}^{k}\right|,\left|J_{x x}^{\infty}-J_{x x}^{k}\right|\right\}<\varepsilon_{2} .
$$

Then we obtain by our assumption (iii) that there exists an $\varepsilon_{3}>0$ such that

$$
g\left(t, x, u^{*}\right)+\mathcal{A} J^{k}\left(t, x, u^{*}\right)>\varepsilon_{3}>0, \quad \forall k \geq k_{0} .
$$

But this is a contradiction to 3.2 which can be seen as follows: By construction we know that a.s.:

$$
\sup _{u \in D}\left\{g\left(s, X_{s}^{k}, u\right)+\mathcal{A} J^{k-1}\left(s, X_{s}^{k}, u\right)\right\} \geq 0 .
$$


Moreover, it follows again from assumption (iii) that there exist $\delta_{1}, \delta_{2}, \delta_{3}>0$ such that for all policies $\pi$

$$
\mathbb{P}_{0, x}\left(X_{s}^{\pi} \in U_{\delta_{1}}(x) \text { for all } 0 \leq s \leq \delta_{2}\right)>\delta_{3} .
$$

Hence the continuity of $(t, x) \mapsto g\left(t, x, u^{*}\right)+\mathcal{A} J^{k}\left(t, x, u^{*}\right)$ together with (i) implies that besides (3.3) the expression is also positive with positive probability for all $k \geq k_{0}$ in a small time interval when we insert the state process $X^{k}$.

The problem can be treated similar when $T$ is replaced by a stopping time $\tau$. Let us now consider a problem with infinite horizon, i.e. we want to maximize

$$
J^{\pi}(t, x):=\mathbb{E}_{t, x}\left[\int_{t}^{\infty} g\left(s, X_{s}, \pi_{s}\right) d s\right], \quad(t, x) \in E .
$$

We assume that

$$
\sup _{\pi \in A(t, x)} \mathbb{E}_{t, x}\left[\int_{t}^{\infty}\left|g\left(s, X_{s}, \pi_{s}\right)\right| d s\right]<\infty .
$$

In principle the same improvement algorithm can be used here. We obtain the following result:

Theorem 3.4. Let $I:=\left\{(t, x) \in E: g(t, x, \hat{\pi}(t, x))+\mathcal{A} J^{\pi}(t, x, \hat{\pi}(t, x))>0\right\}$ and suppose $\hat{\pi}$ satisfies (2.3) for all $T>0$.

a) If $I \neq \emptyset$ and $\liminf _{T \rightarrow \infty} \mathbb{E}_{t, x} J^{\pi}\left(T, \hat{X}_{T}\right) \leq 0$, then $J^{\hat{\pi}}(t, x) \geq J^{\pi}(t, x)$ for all $(t, x) \in E$ and $J^{\hat{\pi}}(t, x)>J^{\pi}(t, x)$ for $(t, x) \in I$.

b) If $I=\emptyset$ and $\lim \sup _{T \rightarrow \infty} \mathbb{E}_{t, x} J^{\pi}\left(T, \tilde{X}_{T}\right) \geq 0$ for all $\tilde{\pi} \in A(t, x)$, then $\pi$ is an optimal control.

Proof. As in the proof of Theorem 3.1 we obtain

$$
J^{\pi}(t, x) \leq \mathbb{E}_{t, x}\left[\int_{t}^{T} g\left(s, \hat{X}_{s}, \hat{\pi}_{s}\right) d s\right]+\mathbb{E}_{t, x}\left[J^{\pi}\left(T, \hat{X}_{T}\right)\right]
$$

for all $T>0$. Taking $\liminf \operatorname{in}_{T \rightarrow \infty}$ we obtain with dominated convergence and our assumption that

$$
J^{\pi}(t, x) \leq \mathbb{E}_{t, x}\left[\int_{t}^{\infty} g\left(s, \hat{X}_{s}, \hat{\pi}_{s}\right) d s\right]=J^{\hat{\pi}}(t, x) .
$$

If $I=\emptyset$, i.e. no improvement is possible, then the proof is as in Theorem 3.1 .

\section{Applications}

In this section we show how the improvement algorithm can be used to prove some theoretical results.

Let us consider the following portfolio optimization problem (for other financial optimization problems see Pham (2009)): The financial market is given by one risky asset and one riskless bond with the following dynamics for $t \in[0, T]$ :

- The price process $\left(S_{t}^{0}\right)$ of the riskless bond is given by

$$
S_{t}^{0}:=e^{r t},
$$

where $r \geq 0$ denotes the fixed continuous interest rate. 
- The price process $\left(S_{t}\right)$ of the risky asset satisfies the stochastic differential equation:

$$
d S_{t}=S_{t-}\left(\mu d t+\sigma d W_{t}+\int_{-1}^{\infty} z \tilde{N}(d t, d z)\right)
$$

where $\mu \in \mathbb{R}, \sigma>0$ are given constants and $\int_{-1}^{\infty} z \nu(d z)<\infty$. The initial price $S_{0}^{k}$ is assumed to be fixed and strictly positive.

Note that this market is free of arbitrage. Further we suppose that our investor has a strictly increasing and strictly concave utility function $U:(0, \infty) \rightarrow \mathbb{R}$ with $U \in C^{2}$, i.e. we have to make sure that $X_{T}^{\pi}>0$ a.s.. Thus, since the market is free of arbitrage, we have to make sure that the wealth process stays positive a.s. which in turn implies that we have to exclude short-sellings and we can define portfolio strategies in terms of invested fractions of the wealth. So in what follows, an admissible portfolio strategy is given by an $\left(\mathcal{F}_{t}\right)$-adapted stochastic process $\left(\pi_{t}\right)$ with values in $D:=[0,1]$ where $\pi_{t}$ gives the fraction of wealth invested in the stock at time $t$. The quantity $1-\pi_{t}$ is the fraction invested in the bond, thus the portfolio is self-financing. Let us denote by $A(t, x)$ the set of all admissible portfolio strategies when we start at time $t \in[0, T]$ with initial capital $x>0$. The dynamics of the wealth process is

$$
d X_{t}^{\pi}=X_{t-}^{\pi}\left(r d t+\pi_{t-} \cdot(\mu-r) d t+\pi_{t-} \sigma d W_{t}+\pi_{t-} \int_{-1}^{\infty} z \tilde{N}(d t, d z)\right) .
$$

The portfolio problem is then to find

$$
J(t, x):=\sup _{\pi \in A(t, x)} \mathbb{E}\left[U\left(X_{T}^{\pi}\right) \mid X_{t}^{\pi}=x\right] .
$$

Note that the problem satisfies the continuity requirements of section 2

The Arrow-Pratt measure of relative risk aversion is defined by

$$
-\frac{x U^{\prime \prime}(x)}{U^{\prime}(x)}
$$

The utility function $U$ is a CRRA-utility function if the Arrow-Pratt measure of relative risk aversion is constant $(\mathrm{CRRA}=$ constant relative risk aversion). For $x>$ 0 , define $x_{\gamma}=\log x$ if $\gamma=0$ and $x_{\gamma}=\frac{1}{\gamma} x^{\gamma}$ if $\gamma<1, \gamma \neq 0$. If $U$ is a CRRA-utility function, then $U(x)=a x_{\gamma}+b$ for appropriate constants $a>0, b \in \mathbb{R}$ and $\gamma<1$ and moreover

$$
-\frac{x U^{\prime \prime}(x)}{U^{\prime}(x)}=1-\gamma .
$$

It is well-known that if $U$ is a CRRA-utility function, then the optimal portfolio strategy is to invest a constant fraction $\pi^{*}$ in the stock (see Øksendal and Sulem (2005), chapter 3). Now we are interested in the following two questions:

- Are there other utility functions where it is optimal to invest a constant fraction of the wealth in the stock?

- When is the strategy "invest all the money in the bond" optimal?

The second problem is rather easy to solve:

Theorem 4.1. Let $U$ be an arbitrary utility function. The "invest all the money in the bond"-strategy is optimal if and only if $\mu \leq r$. 
Proof. Suppose $U$ is an arbitrary utility function. We consider the portfolio strategy $\pi_{t} \equiv 0$. The corresponding value function is obviously $J^{\pi}(t, x)=U\left(x e^{r(T-t)}\right)$. Note that by our assumption $J^{\pi} \in C^{1,2}$. This strategy is optimal if and only if it cannot be improved by the algorithm. For an improvement we have to maximize

$$
u \mapsto \mathcal{A} J^{\pi}(t, x, u), \quad u \in[0,1]
$$

where the generator is given by

$$
\begin{aligned}
\mathcal{A} J^{\pi}(t, x, u)= & J_{t}^{\pi}+J_{x}^{\pi} x(r+u(\mu-r))+\frac{1}{2} J_{x x}^{\pi} x^{2} u^{2} \sigma^{2}+ \\
& +\int_{-1}^{\infty}\left(J^{\pi}(t, x+u x z)-J^{\pi}(t, x)-J_{x}^{\pi}(t, x) u x z\right) \nu(d z) .
\end{aligned}
$$

Obviously $u \mapsto \mathcal{A} J^{\pi}(t, x, u)$ is concave. Thus $\pi^{*} \equiv 0$ is again a maximizer if and only if

$$
\left.\frac{\partial}{\partial u} \mathcal{A} J^{\pi}(t, x, u)\right|_{u=0} \leq 0 .
$$

Differentiating we obtain:

$$
\begin{aligned}
\frac{\partial}{\partial u} \mathcal{A} J^{\pi}(t, x, u)= & (\mu-r) x J_{x}^{\pi}+\sigma^{2} x^{2} u J_{x x}^{\pi}+ \\
& +\int_{-1}^{\infty}\left(J_{x}^{\pi}(t, x+u x z) x z-J_{x}^{\pi}(t, x) x z\right) \nu(d z)
\end{aligned}
$$

and 4.2 is satisfied if and only if $(\mu-r) x J_{x}^{\pi} \leq 0$. Since $x J_{x}^{\pi} \geq 0$ it must hold that $\mu \leq r$.

Remark 4.2. Suppose now we have a Black-Scholes market, i.e. there are no jumps in the stock price. In case $\mu \neq r$, the first improvement of the "invest all the money in the bond"-strategy is then given by

$$
\hat{\pi}(t, x)=-\frac{U^{\prime}\left(x e^{r(T-t)}\right)}{U^{\prime \prime}\left(x e^{r(T-t)}\right) x e^{r(T-t)}} \cdot \frac{(\mu-r)}{\sigma^{2}} .
$$

It relies on the Arrow-Pratt measure of relative risk aversion

$$
-\frac{U^{\prime \prime}\left(x e^{r(T-t)}\right) x e^{r(T-t)}}{U^{\prime}\left(x e^{r(T-t)}\right)} \text { and } \frac{(\mu-r)}{\sigma^{2}}
$$

which is the Merton-ratio. When the utility function a CRRA-utility function, then

$$
-\frac{U^{\prime}\left(x e^{r(T-t)}\right)}{U^{\prime \prime}\left(x e^{r(T-t)}\right) x e^{r(T-t)}}=\frac{1}{1-\gamma}
$$

and the first improvement yields already the optimal investment strategy.

The answer to the first question is a little bit more demanding. Let us therefore consider the special case that the Lévy measure is concentrated on $(0, \infty)$, i.e. jumps are only upwards and that $2 \int x \nu(d x)<\mu-r$. In this case we can allow $D:=[0, \infty)$. Under these assumptions the answer to the first question is given in the next theorem:

Theorem 4.3. The CRRA-utility functions are the only utility functions where the optimal portfolio invests a constant positive fraction of the wealth in the stock. 
Proof. Suppose $U$ is an arbitrary utility function. We consider the portfolio strategy which invests the constant fraction $\pi_{t} \equiv \pi>0$ in the stock and denote the corresponding value function by $J^{\pi}$. Note that

$$
\begin{gathered}
J^{\pi}(t, x)=\mathbb{E} U\left(x \operatorname { e x p } \left(\left(r+(\mu-r) \pi-\frac{1}{2} \pi^{2} \sigma^{2}\right)(T-t)+\sigma \pi W_{T-t}+\right.\right. \\
\left.\left.\int_{t}^{T} \int \log (1+\pi y) \tilde{N}(d s, d y)\right)\right)
\end{gathered}
$$

and hence $J^{\pi} \in C^{1,2}$. Further it follows directly that $x \mapsto J^{\pi}(t, x)$ is strictly increasing and strictly concave. Thus in particular $J_{x x}^{\pi} \leq 0$ and the mapping $u \mapsto \mathcal{A} J^{\pi}(t, x, u)$ is concave. Hence the strategy $\pi_{t} \equiv \pi$ is optimal if and only if it cannot be improved by the algorithm. This means that $J^{\pi}$ and $\pi$ are optimal if and only if $\pi$ is a maximum point of $u \mapsto \mathcal{A} J^{\pi}(t, x, u), u \geq 0$, i.e.

$$
(\mu-r) J_{x}^{\pi}+J_{x x}^{\pi} \sigma^{2} x \pi+\int_{0}^{\infty}\left(J_{x}^{\pi}(t, x+\pi x z) z-J_{x}^{\pi}(t, x) z\right) \nu(d z)=0
$$

and we must have $\mathcal{A} J^{\pi}(t, x, \pi)=0$, i.e.

$$
\begin{aligned}
& J_{t}^{\pi}+(r+(\mu-r) \pi) x J_{x}^{\pi}+\frac{1}{2} J_{x x}^{\pi} \sigma^{2} x^{2} \pi^{2}+ \\
& +\int_{0}^{\infty}\left(J^{\pi}(t, x+\pi x z)-J^{\pi}(t, x)-J_{x}^{\pi}(t, x) \pi x z\right) \nu(d z)=0 .
\end{aligned}
$$

Equation (4.3) is independent of $t$, i.e. we may set $h(x)=J_{x}^{\pi}(t, x)$ and obtain

$$
h^{\prime}(x)+\frac{\mu-r-\int z \nu(d z)}{\sigma^{2} x \pi} h(x)=-\frac{1}{\sigma^{2} x \pi} \int_{0}^{\infty}(h(x+\pi x z) z) \nu(d z)
$$

We claim now that under our assumption the integro-differential equation 4.5 has a unique bounded solution on $[\varepsilon, \infty)$ for arbitrary $\varepsilon>0$ with initial value $h(\varepsilon)=h_{0}$. This statement is shown in the Appendix. Now depending on the precise value of $\pi>0$, the solution of 4.5 (up to constants) can have two different forms. It can either be

$$
h(x)=x^{\gamma-1}, \quad \gamma<1, \gamma \neq 0
$$

in which case $\pi$ is the solution of

$$
f(\pi ; \gamma)=\left(\mu-r-\int z \nu(d z)\right)+(\gamma-1) \sigma^{2} \pi+\int_{0}^{\infty} z(1+\pi z)^{\gamma-1} \nu(d z)=0 .
$$

Note that this equation has a unique solution $\pi(\gamma)$ on $(0, \infty)$, since $f(0 ; \gamma)=\mu-r>$ 0 and $\pi \mapsto f(\pi ; \gamma)$ is continuous and decreasing to $-\infty$. The other possible solution is

$$
h(x)=\frac{1}{x}
$$

in which case $\pi$ is the solution $\pi(0)$ of $f(\pi ; 0)=0$. Moreover, it is possible to show that $\gamma \mapsto f(\pi ; \gamma)$ is strictly increasing (as long as $\pi \neq 0$ ) which implies that $\gamma \mapsto \pi(\gamma)$ is strictly increasing. In total we know that every $\pi \in(0, \infty)$ is the zero of exactly one $f(\pi ; \gamma)$ which identifies $\gamma$. The functions $f(\pi ; \gamma)$ are plotted for $\gamma=-0.5,0,0.5$ and parameters $\mu-r=0.1, \int z \nu(d z)=\frac{1}{2}, \sigma^{2}=0.04, \nu=\operatorname{Unif}(0,1)$ in figure 1 .

Thus, the possible solutions for $J^{\pi}$ are given by

(a) $J^{\pi}(t, x)=\frac{a(t)}{\gamma} x^{\gamma}+b(t), \quad a(t)>0, t \in[0, T]$.

(b) $J^{\pi}(t, x)=a(t) \log x+b(t), \quad a(t)>0, t \in[0, T]$. 


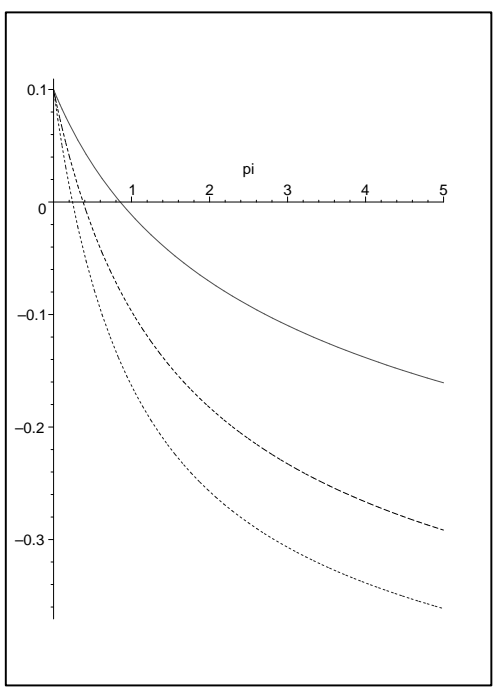

FIgURE 1. Function $\pi \mapsto f(\pi, \gamma)$ for $\gamma=0.5$ (upper line), $\gamma=0$ and $\gamma=-0.5$ (lower line).

Inserting these expressions into equation 4.4 yields in the first case $b(t) \equiv b$ and $a(t)=\exp \left(-\left(\gamma(r+(\mu-r) \pi)+\frac{1}{2} \gamma(\gamma-1) \sigma^{2} \pi^{2}+\int_{0}^{\infty}(1+\pi z)^{\gamma}-(1+\gamma \pi z) \nu(d z)\right) t\right)$.

In the second case we obtain $a(t) \equiv a$ and

$$
b(t)=a\left(\frac{1}{2} \sigma^{2} \pi^{2}-r-(\mu-r) \pi+\int z \pi-\log (1+\pi z) \nu(d z)\right) t+b, b \in \mathbb{R} .
$$

Both solutions are unique up to a multiplicative constant which proves the statement.

Remark 4.4. From the proof of Theorem 4.3 it follows that the optimal portfolio fraction $\pi^{*}=\pi^{*}(\gamma)$ is strictly increasing in $\gamma$, where $1-\gamma$ is the Arrow-Pratt measure of relative risk aversion of the utility function, i.e. the more risk averse the investor is, the less she invests into the stock.

Remark 4.5. Note that the Black-Scholes market (i.e. there are no jumps in the stock price process) is a special case of our model and hence Theorem 4.3 holds true in this case.

\section{ApPENDix}

Proposition 5.1. The integro-differential equation (4.5) has a unique bounded solution on $[\varepsilon, \infty)$ for arbitrary $\varepsilon>0$ with initial value $h(\varepsilon)=h_{0}>0$. 
Proof. Let $\mathbb{B}:=\{v:[\varepsilon, \infty) \rightarrow \mathbb{R}: v$ is measurable and bounded by a constant $\}$ and for $v \in \mathbb{B}$ let $\|\cdot\|$ be the supremum-norm. On $\mathbb{B}$ we define the operator $\mathbb{T}$ by: $\mathbb{T} \hat{h}$ is the (unique) solution of the linear differential equation

$$
h^{\prime}(x)+\frac{\mu-r-\int z \nu(d z)}{\sigma^{2} x \pi} h(x)=-\frac{1}{\sigma^{2} x \pi} \int_{0}^{\infty}(\hat{h}(x+\pi x z) z) \nu(d z)
$$

on $[\varepsilon, \infty)$ with $h(\varepsilon)=h_{0}$. The unique solution of this initial value problem is given by (where we denote $\kappa:=\frac{\mu-r-\int z \nu(d z)}{\sigma^{2} \pi}>0$ ):

$$
\mathbb{T} \hat{h}(x)=h_{0}\left(\frac{\varepsilon}{x}\right)^{\kappa}-\frac{1}{x^{\kappa}} \frac{1}{\sigma^{2} \pi} \int_{\varepsilon}^{x} \int_{0}^{\infty} \hat{h}(t(1+\pi z)) z \nu(d z) t^{\kappa-1} d t .
$$

If $\|\hat{h}\| \leq L$ then

$$
\|\mathbb{T} \hat{h}\| \leq L
$$

hence $\mathbb{T}: \mathbb{B} \rightarrow \mathbb{B}$ and $\mathbb{B}$ is a Banach space together with the supremum norm. Finally it is possible to show that $\mathbb{T}$ is also contracting. This follows since by a similar calculation as above we obtain:

$$
\|\mathbb{T} \hat{h}-\mathbb{T} h\| \leq\|\hat{h}-h\| \frac{\int z \nu(d z)}{\mu-r-\int z \nu(d z)}
$$

where $\frac{\int z \nu(d z)}{\mu-r-\int z \nu(d z)}<1$ due to our assumption. Thus according to Banach's fixed point theorem there exists a unique solution of the initial value problem.

\section{REFERENCES}

Fleming, W. H. and Rishel, R. W. (1975) Deterministic and stochastic optimal control. Springer-Verlag, New York. 1

Fleming, W. H. and Soner, H. M. (1993) Controlled Markov processes and viscosity solutions, volume 25 of Applications of Mathematics (New York). SpringerVerlag, New York. 1

Howard, R. A. (1960) Dynamic programming and Markov processes. The Technology Press of M.I.T., Cambridge, Mass. 1

Kushner, H. J. and Dupuis, P. (2001) Numerical methods for stochastic control problems in continuous time, volume 24 of Applications of Mathematics (New York). Springer-Verlag, New York, second edition. 1

Ørsendal, B. and Sulem, A. (2005) Applied stochastic control of jump diffusions. Universitext. Springer-Verlag, Berlin. 1, 2, 4, 7

Pham, H. (2009) Continuous-time stochastic control and optimization with financial applications, volume 61 of Stochastic Modelling and Applied Probability. Springer-Verlag, Berlin. 6

Schmidui, H. (2008) Stochastic control in insurance. Probability and its Applications. Springer, London. 1

Yong, J. and Zhou, X. Y. (1999) Stochastic controls, volume 43 of Applications of Mathematics (New York). Springer-Verlag, New York. 1 
(N. Bäuerle) Institute for Stochastics, Karlsruhe Institute of Technology, D-76128 Karlsruhe, Germany

E-mail address: nicole.baeuerle@kit.edu

(U. Rieder) Department of Optimization and Operations Research, University of Ulm, D-89069 Ulm, Germany

E-mail address: ulrich.rieder@uni-ulm.de 